\title{
Dilemas del perito experto: derechos indígenas a la tierra en Surinam y Guyana*
}

\author{
Stuart Kirsch
}

Universidad de Michigan, Estados Unidos

skirsch@umich.edu

Publicado en Desacatos 57, mayo-agosto 2018, doi: https://doi. org/10.29340/57.1949

-Traducido por Luis Manuel Claps

\section{Resumen}

Este artículo examina dos informes periciales presentados ante la Comisión y la Corte Interamericana de Derechos Humanos. El primero, sobre la negativa de Surinam a reconocer los derechos a la tierra de los pueblos indígenas, a pesar de sus obligaciones internacionales. El segundo, sobre títulos de tierras de población indígena en Guyana. Comparar ambos casos me permite hacer observaciones sobre la investigación etnográfica a corto plazo para peritajes antropológicos, incluyendo la necesidad de hacer que estos informes resulten comprensibles en tres marcos superpuestos: el de los abogados y el sistema legal, el de las comunidades en lucha por sus derechos, y el de la disciplina antropológica. Además, considero las elecciones narrativas adoptadas en los informes, los dilemas políticos de ser perito experto y los desafíos de la etnografía a corto plazo.

Palabras clave: antropología legal, derechos indígenas, perito experto, Corte Interamericana de Derechos Humanos, antropología comprometida

En agosto de 2014, visité la aldea de Isseneru en el río Mazaruni medio, en Guyana. El abogado de la comunidad me pidió que realizara una investigación y escribiera un informe de testigo experto para la Comisión Interamericana de Derechos Humanos sobre los problemas asociados a su título de tierras otorgado por el Estado en 2007. Durante una reunión preliminar para discutir el proyecto, les expliqué que el Estado había argumentado que Isseneru "no [era] una comunidad tradicional akawaio" porque su población se relacionaba con 
extranjeros, hablaba inglés, se dedicaba a la minería de oro artesanal y recibía un porcentaje del oro que trabajadores mineros no indígenas extraían de sus tierras (Government of Guyana, 2013: 11, 22). El Estado también rechazó su reclamo de ser los propietarios tradicionales de Isseneru, por haber sido relocalizados allí desde el Mazaruni alto, en la década de 1970 (2013: 11, 22).

Como era previsible, los asistentes a la reunión reaccionaron contra mis argumentos de manera enérgica. Dijeron que estaban "extremadamente molestos por escuchar lo que de ellos se decía”. También objetaron que se les pidiera que se explicaran a sí mismos, diciendo "otro visitante quiere saber quiénes somos", y mencionaron que habían sido interrogados con anterioridad sobre "cómo vivimos, de dónde venimos, de quién obtuvimos nuestro título de propiedad". Agregaron: "sabemos que esta tierra es nuestra, por eso vivimos en ella" y "nuestros abuelos [y ancestros] vivieron, cultivaron y cazaron en todas estas tierras". Un poco avergonzado por su respuesta, reconocí la validez de sus argumentos y repetí que estaba allí para ayudar a documentar la demanda legal por la tierra, no para juzgar sus decisiones.

El presente artículo se vincula a dos informes de testigo experto presentados ante la Comisión y la Corte Interamericana de Derechos Humanos (cidh), referidos a los derechos indígenas a la tierra en los países vecinos de Surinam y Guyana. El primero, sobre los perjuicios ocasionados por la negativa de Surinam a reconocer los derechos a la tierra de los pueblos indígenas y así omitir sus obligaciones internacionales. El segundo, acerca de los problemas asociados a la tenencia indígena de la tierra en Guyana, bajo la Ley Amerindia de 2006. Las solicitudes de participación en estos proyectos llegaron de Fergus MacKay, consejero de la organización Forest Peoples Programme, en Reino Unido, quien patrocinó las demandas indígenas en ambos casos. En una ocasión anterior, MacKay me había pedido que contribuyera a la revisión independiente de un proyecto minero de bauxita, ubicado en las montañas Bakhuis, al oeste de Surinam (Goodland, 2009; Kirsch, 2009). Antes de aceptar, tuve oportunidad de leer varios reportes, informes periciales y fallos preliminares, y discutir con MacKay sobre los procesos legales. Él no dio indicaciones sobre la forma ni el contenido de los informes de testigo experto, aunque sugirió algunas preguntas $\mathrm{y}$ aportó comentarios a versiones preliminares.

A continuación, presento resúmenes de los informes de testigo experto expuestos en los dos casos. El lector verá que describo de manera estructural los dos sistemas de tenencia, en lugar de examinar cómo las prácticas reales se alejan de esos principios. Esto refleja las dificultades que supone la etnografía a corto plazo que se realiza con el propósito de escribir informes de experto. Resulta también de la necesidad de asegurar que los informes sean legibles para tres audiencias distintas, cada una con su propio marco de referencia: los abogados y el sistema legal, las comunidades en lucha por sus derechos y la disciplina antropológica (Paine, 1996: 63), como discuto más adelante. 


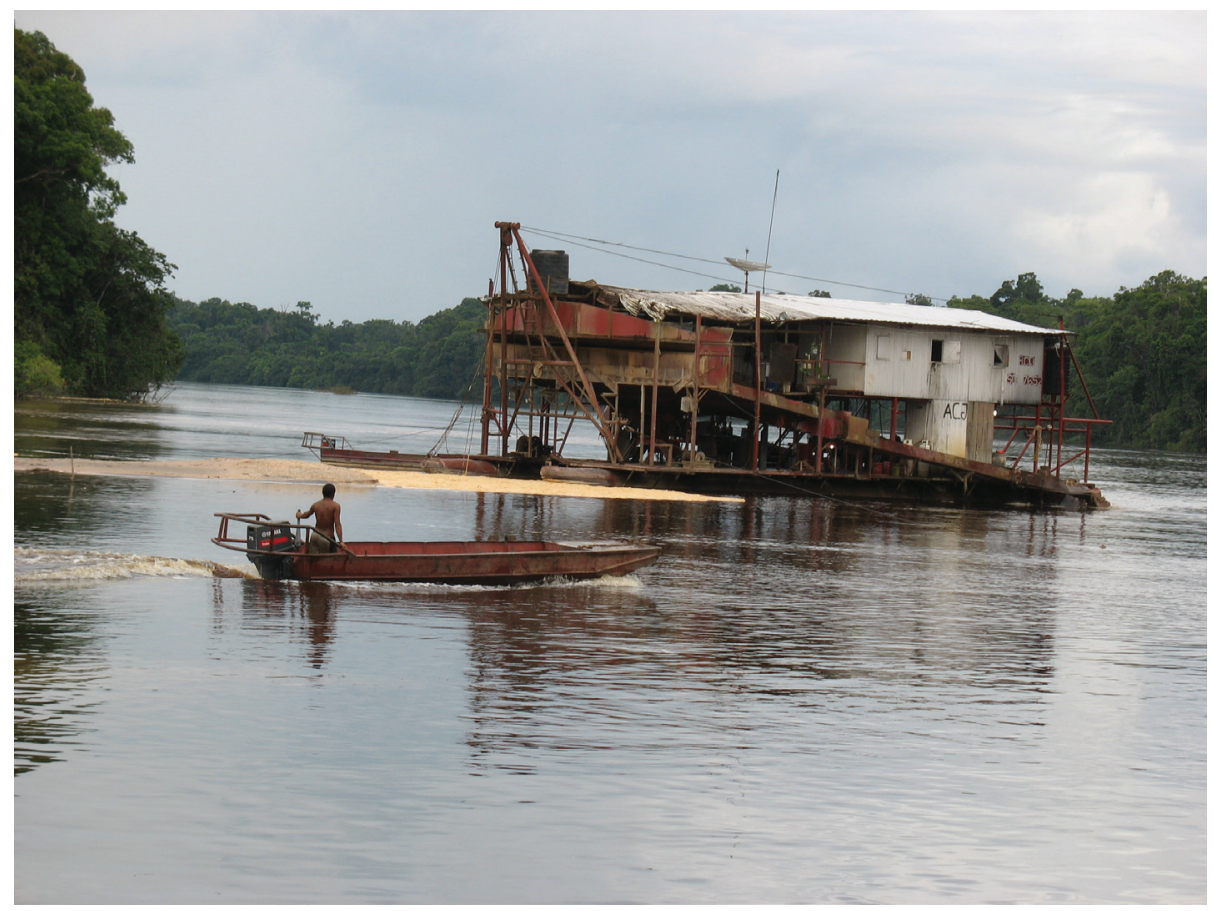

Figura 1. Dragas mineras operadas por foráneos en el río Mazaruni medio, cerca de la aldea Isseneru, Guyana.

Además, hago una breve descripción de las audiencias y la sentencia del caso de Surinam. El veredicto del caso de Guyana continúa pendiente. Por último, examino las elecciones narrativas que consideré al escribir estos textos, los dilemas políticos asociados a los informes de testigo experto y los límites de la investigación basada en etnografía a corto plazo. El objetivo del artículo es discutir algunos de los procesos involucrados en la producción de informes externos por antropólogos, lo que incluye interacciones con comunidades y su influencia en los procedimientos legales, tanto en los hallazgos del investigador como en la forma en que son presentados, así como si pueden ser considerados etnografía "suficiente" (Scheper-Hughes, 1989: 28).

\section{Caso 1: derechos indígenas a la tierra en Surinam}

El primer caso examina las consecuencias negativas del rechazo estatal a reconocer los derechos indígenas a la tierra de los pueblos kaliña y lokono, en la región del Marowijne inferior, en Surinam. Sus territorios fueron progresivamente reducidos y degradados por la minería, la industria forestal y la expansión de la ciudad de Albina hacia las tierras indígenas. Además, el Estado 
estableció varias reservas naturales en sus territorios sin su consentimiento. Este caso da continuidad a los esfuerzos de los saramaka maroons por obligar al Estado a reconocer sus derechos a la tierra, quienes ya lograron un fallo que sentó precedente en la Corte Interamericana de Derechos Humanos (cidh, 2008). Sin embargo, desde entonces el Estado no ha implementado la decisión ni reconocido los derechos a la tierra tanto de los maroons, que representan más de $20 \%$ de la población, como de los pueblos amerindios, una pequeña minoría en Surinam.

La Reserva Natural Galibi fue establecida por las autoridades de la Colonia holandesa en 1969, con el fin de proteger la zona costera en la que cada año las tortugas de mar, entre ellas varias especies en peligro de extinción, llegan a depositar sus huevos. Ninguno de los capitanes o líderes locales elegidos fue capaz de leer los documentos que se les pidió que firmaran y pensaron que estaban autorizando un proyecto de investigación. Algunas viviendas y cultivos agrícolas quedaron ubicados dentro de los límites de la reserva y fueron obligados a reubicarse. Si bien varios integrantes de la comunidad habían desarrollado con éxito emprendimientos ecoturísticos que operaban durante la temporada de anidación, no recibieron una compensación adecuada por sus tierras. En un primer momento, albergaron la esperanza de que el gobierno posindependentista de Surinam reconociera sus derechos a la tierra y los recursos, pero el Estado persistió en su negativa. Desde la década de 1970, los kaliña han demandado de manera consistente la restitución de todas las tierras incorporadas a la Reserva Galibi y siguen sintiéndose agraviados porque una parte integral de su territorio fue despojada de manera unilateral.

Los capitanes de las aldeas de Galibi también creen que el Estado no protege a las poblaciones de tortugas marinas, algunas de las cuales están declinando. Argumentan que la amenaza más seria para ellas es quedar atrapadas y morir por ahogamiento en las extensas redes que los barcos pesqueros despliegan en el estuario del río Marowijne. La mayoría de estos barcos viene de la capital Paramaribo, pero el Estado ejerce muy poco control sobre ellos (Kambel, 2002: 143). La Reserva Natural Galibi sigue imponiéndoles estrictas reglas de conservación, a pesar de su tradicional tabú a la ingesta de tortugas, aunque en el pasado hayan cosechado sus huevos para consumo y venta. Como me dijo Ricardo Pané, capitán de Galibi:

No necesitamos una reserva natural para proteger a los animales, porque contamos con conocimientos tradicionales muy antiguos para hacerlo. Aunque el gobierno no ha logrado proteger la biodiversidad [ . . . ], los pueblos indígenas la conservan incluso antes de la llegada de los conquistadores españoles a América del Sur (entrevista con Ricardo Pané, Galibi, Surinam, 19 de enero de 2009). 
El capitán Pané se preguntaba por qué las tortugas marinas gozaban de derechos a la tierra, pero no el pueblo indígena de Surinam.

El establecimiento de la Reserva Natural Wane Kreek, en 1986, implicó una nueva toma de tierras indígenas en el Marowijne inferior (vids, 2009: 49). Esta acción provocó más consecuencias destructivas que la Reserva Galibi, en la cual la tierra está protegida del desarrollo. Por el contrario, Wane Kreek se convirtió en zona industrial para la extracción de recursos, con una mina de bauxita operada por Suralco y bhp Billiton.

A finales de la década de 1970, las autoridades coloniales holandesas encargaron un inventario de los ecosistemas costeros del país. Por el intenso desarrollo a lo largo de la costa y el comparativamente intacto ecosistema de Wane Kreek, el área fue designada reserva natural a pesar de la preexistencia de derechos mineros otorgados por las autoridades coloniales holandesas a la empresa Suralco, subsidiaria de Aluminum Company of America (Alcoa), así como la existencia de concesiones forestales anteriores. Los pueblos kaliña y lokono están concientes de la ironía que implica que el Estado haya expropiado las tierras indígenas para establecer un área de conservación - precisamente porque la sustentabilidad de esas comunidades manejó los recursos del lugary luego permitiera su conversión en zona extractiva.

En el pasado, los pueblos indígenas del Marowijne inferior cazaron, pescaron y acamparon en Wane Kreek y las sierras adyacentes con el mismo nombre. La gente que vive allí comentó: "no necesitamos reservas naturales para proteger la biodiversidad", porque ya cuentan con reglas, tabúes y límites de consumo y extracción que "en la práctica conducen a la protección del área, los animales y las especies de plantas" (clim, 2006: 113). Como una persona explicó:

Somos pueblos de la naturaleza, vivimos en ella y por ella. Pero las personas que tomaron la decisión [de establecer la reserva natural] no viven en la naturaleza, ellos trabajan en oficinas y toman decisiones sin nosotros. Utilizan los conocimientos de los pueblos indígenas [para la conservación del bosque] en contra de los pueblos indígenas [al establecer una reserva natural en nuestras tierras] (entrevista con informante, Wan Shi Sha, aldea Marijkedorp, Surinam, 18 de enero de 2009).

Cuando Suralco comenzó a extraer bauxita en Wane Hills, a mediados de la década de 1990, se hizo evidente que la Reserva Natural Wane Kreek resultaría afectada. Los yacimientos de bauxita se encuentran, por lo general, cerca de la superficie terrestre, de modo que la extracción del mineral se efectúa típicamente mediante la minería a cielo abierto de extensiones kilométricas. Una significativa porción de la cubierta forestal de la reserva fue talada y la fauna que la habitaba fue desplazada. La capa de laterita roja que queda después de la extracción de bauxita es hostil a la regeneración del bosque y los 
esfuerzos de la empresa por rehabilitarlos exhibieron una eficacia limitada. Aunque la minería de bauxita en Wane Hills está por acabarse, los anchos caminos construidos por la empresa minera abrieron el área a otras actividades extractivas, incluyendo la tala legal e ilegal, la extracción de arena y grava, y más recientemente, la minería de caolín. Las tierras despojadas a los pueblos indígenas para establecer la reserva natural se convirtieron de facto en un sistema de libre acceso. ${ }^{1}$

Los kaliña y los lokono también dan testimonio del impacto del desarrollo y la degradación ambiental en sus prácticas de subsistencia, porque los obligan a participar de manera más intensa en la economía monetaria. Como me dijo una persona: "antes se podía vivir sin dinero, pero ahora lo necesitamos [para sobrevivir]". Ya no son capaces de alimentar a sus familias mediante la caza, la pesca y la agricultura. Un líder comunitario me dijo que participar en la economía monetaria está bien para quienes son exitosos, pero no todos los integrantes de la comunidad cuentan con las habilidades y capacidades necesarias para ganarse un salario. Además, comparten los alimentos entre ellos cuando cazan o pescan, pero no cuando los compran con dinero. El fracaso del Estado en reconocer los derechos indígenas a la tierra los obligó a enfrentar nuevas formas de inequidad estructural.

Los kaliña y los lokono describen cómo estos cambios económicos y medioambientales también afectaron sus posibilidades de reproducir la propia cultura. Muchas de las habilidades prácticas asociadas a la producción de subsistencia ya no se enseñan de padres a hijos ni de madres a hijas: "en algunas familias ya no hay ancianos que les enseñen estas cosas. Incluso para conseguir los materiales necesarios [ . . . ], ya no se encuentran en la localidad, por la tala, hay que trasladarse grandes distancias para conseguirlos" (entrevista con informante, Wan Shi Sha, aldea Marijkedorp, Surinam, 18 de enero de 2009). Sin embargo, nuevos mercados para productos amerindios, como el pan y la cerveza de yuca, conocida como kasiri, mercancías agrícolas y frutos exóticos, han aparecido en Surinam y la ciudad de Saint-Laurent-du-Maroni, cruzando el río desde Albina, en la Guayana Francesa. Participar en estos mercados ofrece a los kaliña y lokono la oportunidad de mejorar sus estándares de vida al utilizar habilidades y conocimientos tradicionales. Sin embargo, la constante variabilidad de estas prácticas, debido al avance de la degradación ambiental provocada por la minería y la industria forestal en sus tierras, los expone a riesgos considerables.

En grupos focales y entrevistas, el elemento destacado de nuestra discusión sobre la importancia de los derechos indígenas a la tierra fue su invocación de la libertad. La gente me dijo que sólo se siente libre en su propia tierra, donde puede

1. Feeny et al. definen acceso abierto como "la ausencia de derechos de propiedad bien definidos. El acceso a los recursos es desregulado, gratuito y abierto a cualquiera. Muchas zonas de pesca lejos de la costa antes del siglo xx o la atmósfera sirven de ejemplo" (1990: 4). 
hacer lo que le plazca. Sin derechos a la tierra, subrayaron, uno no es realmente libre, porque "cualquiera puede aparecer con un pedazo de papel diciendo que es suya". Muchas personas describieron la libertad como el hecho de poder cazar y pescar en los bosques. Cuando conversé con un grupo de hombres jóvenes sobre su futuro, me dijeron que querían quedarse en su propia tierra porque: "amamos este lugar. Queremos nuestro propio lugar donde poder vivir. Queremos ser libres". Hoy, sin embargo, no son "suficientemente libres [porque] otra gente está viniendo a nuestro territorio". Al describir la reserva natural establecida en sus tierras, expresan sus críticas en términos de restricciones a la libertad: "antes éramos libres de ir y venir, pero ahora alguien nos impone reglas". Muchos recordaron historias sobre la colocación de carteles de "prohibido pasar" sobre las viviendas construidas en tierras enajenadas a los pueblos indígenas. ${ }^{2}$

En febrero de 2015, asistí a las audiencias de la Corte Interamericana de Derechos Humanos sobre el caso kaliña y lokono en San José, Costa Rica. Los capitanes Ricardo Pané y Jona Gunther testificaron ante la Corte sobre las consecuencias del no reconocimiento de los derechos indígenas a la tierra por parte del Estado. El capitán Pané explicó: "estamos aquí para continuar una lucha justa hasta obtener el reconocimiento legal de nuestros derechos a la tierra". En respuesta a una pregunta formulada por el representante del Estado, Pané relató que padecieron sufrimientos físicos y emocionales que les provocaron trauma. Cuando el juez Vio Grossi le preguntó: “ ¿se siente usted un nacional surinamés?", el capitán Pané respondió: "no me siento tal porque mis derechos no han sido respetados". Cuando el juez le preguntó si se sentía ciudadano de Surinam, él respondió: "no".

Jérémie Gilbert, profesor de Leyes de la Universidad de East London y experto en derechos indígenas, testificó que los derechos indígenas a la tierra son plenamente compatibles con la conservación de la biodiversidad (Colchester, 2004). Las mejores prácticas en casos de desalojo de pueblos indígenas por reservas naturales, observó Gilbert, involucran la restitución de sus tierras y subsiguiente negociación de planes de comanejo para la protección de ambientes degradados y especies amenazadas (achpr, 2009). La relatora especial de la Organización de Naciones Unidas sobre los Derechos de los Pueblos Indígenas, Victoria Tauli-Corpuz, también aportó su testimonio sobre las contribuciones de los pueblos indígenas a la conservación de la biodiversidad. En su recapitulación final, el abogado de la parte acusadora, Fergus MacKay, argumentó que la falta de reconocimiento del Estado de la "personería jurídica" de los pueblos indígenas y el no establecimiento de un marco para entregarles los títulos de sus tierras constituían una violación de sus derechos humanos y una contravención de las obligaciones del Estado, según lo dispuesto por diversos tratados y acuerdos internacionales.

2. Comentarios del grupo focal en la aldea Alfonsdorp, Surinam, 20 de enero de 2009. 
La respuesta del Estado a estas acusaciones fue que no se encontraba preparado para implementar nuevas leyes que reconocieran los derechos indígenas a la tierra, ya que podrían exacerbar las tensiones políticas existentes entre los grupos étnicos de Surinam. Durante el receso, uno de los abogados oficiales me dijo que era posible que perdieran el caso, pero que al menos esperaba "ganar tiempo" hasta que el Estado fuera capaz de atender estos temas. Los jueces de la cidh, sin embargo, expresaron su frustración por el fracaso de Surinam en hacer realidad la decisión del caso del pueblo saramaka $v s$. Surinam (cidh, 2008), en la que instruyó al Estado para que reconociera de manera formal los derechos a la tierra de los saramaka maroons. ${ }^{3}$

El 25 de noviembre de 2015, la cidh determinó que el no reconocimiento por parte del Estado de Surinam de la personería jurídica y los derechos territoriales de los pueblos kaliña y lokono, incluyendo sus derechos a la propiedad colectiva, derechos políticos y protección judicial, constituía una violación de la Convención Americana de Derechos Humanos (cidh, 2015). La Corte instruyó al Estado para que garantizara a los kaliña y lokono el título colectivo de sus territorios tradicionales, junto con los recursos necesarios para su demarcación. Esto contemplaba la restitución de tierras indebidamente entregadas a terceros no indígenas. Además, la Corte instruyó al Estado para que diera los pasos necesarios para que los kaliña y lokono contaran con acceso a sus tierras e hicieran uso de ellas en las reservas naturales Galibi y Wane Kreek, que participaran del manejo y aseguraran que las actividades desarrolladas en dichas áreas no provocaran impactos negativos en la población. La Corte también halló violaciones activas a sus derechos en relación con la minería de bauxita desarrollada por empresas subsidiarias de Alcoa y bhp Billiton, sin participación de los kaliña y lokono, toda vez que ésta se desarrolla en territorios indígenas que además son reserva natural. En consecuencia, la Corte ordenó al Estado que rehabilitara la zona afectada por la minería en la Reserva Natural Wane Kreek (cidh, 2015).

El fallo también buscó asegurar que estos hechos no se repitieran al determinar que la decisión se aplicara a todos los pueblos indígenas y tribales de Surinam y no sólo a los del Marowijne inferior (cidh, 2015). Con ello, reafirma los reclamos y solicitudes de los demandantes en casi todas las instancias, pero dado el fracaso del Estado en dar respuesta efectiva a la decisión del caso del pueblo saramaka vs. Surinam (cidh, 2008), habrá que ver hasta dónde se instrumentará el nuevo fallo. 


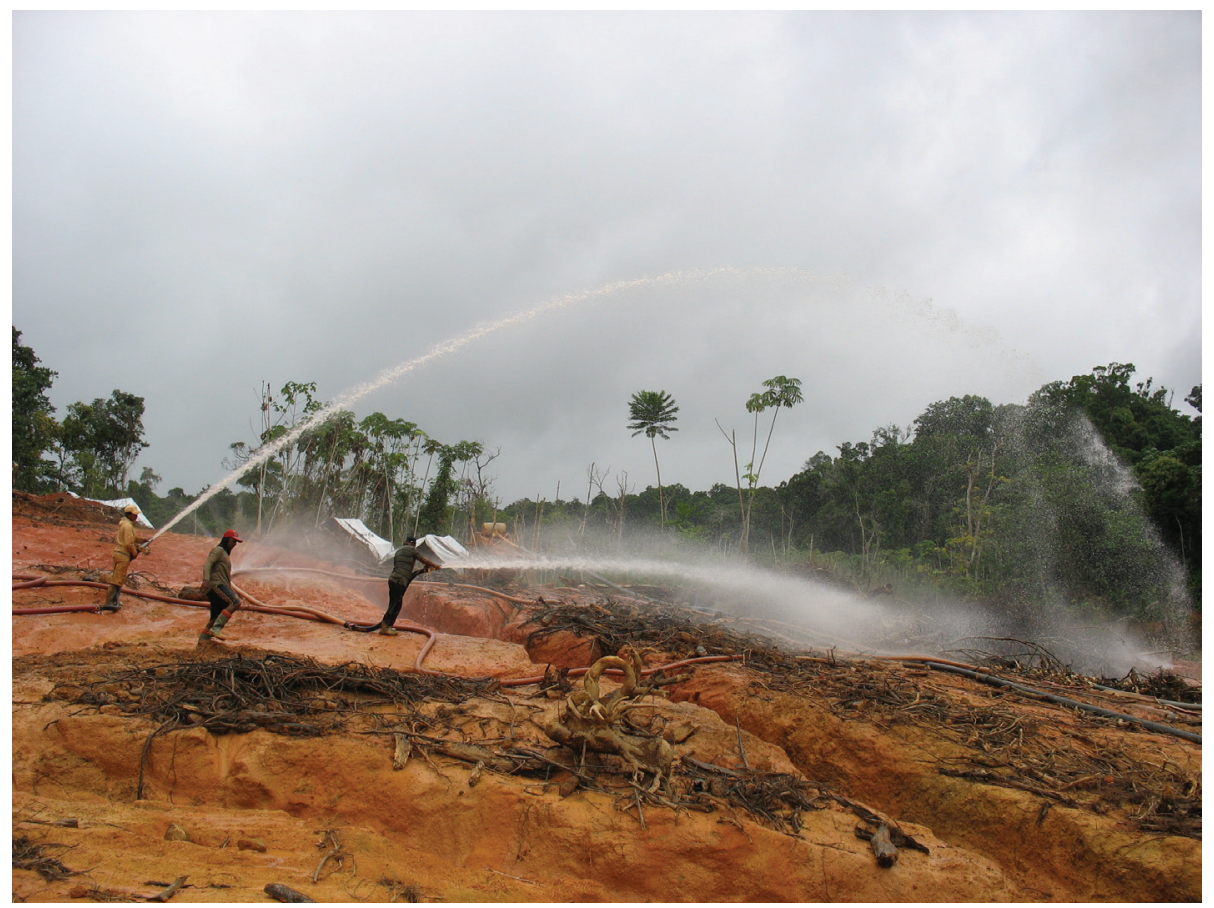

Figura 2. Minería hidráulica utilizada por habitantes de la aldea Isseneru, Guyana.

\section{Caso 2: derechos indígenas a la tierra en Guyana}

En noviembre de 2014, visité Guyana para elaborar un informe de testigo experto sobre el título de tierras otorgado por el Estado al pueblo akawaio de la aldea Isseneru. El área fue invadida por mineros de oro y diamantes que utilizan tecnologías de pequeña escala destructivas para el ambiente, que incluyen el uso de dragas y mercurio para amalgamar el oro. La comunidad indígena también hace minería de oro para apoyar su propia economía, lo cual, como crítico de los impactos ambientales de la minería, generó algunas preguntas difíciles (Kirsch, 2014). La participación indígena en la industria minera también es una forma de competencia desde el punto de vista de los intereses económicos de la población costeña (Hilson y Laing, 2017a). Por esta situación, se percibe que $10 \%$ de la población del país reclama una porción de tierras y recursos nacionales mayor a la que le corresponde.

En 2007, el Estado otorgó el título de unos $260 \mathrm{~km}^{2}$ de tierras a Isseneru. Esta decisión se tomó después de ignorar dos solicitudes previas, de 1987 y 1994. Preocupados por la proliferación de mineros de oro y diamantes que operan dragas en el río Mazaruni, los habitantes de la aldea hicieron otro intento infructuoso de obtener el título de sus tierras en 2003. Tras la aprobación 
de la Ley Amerindia, en 2006, el Ministerio de Asuntos Amerindios invitó de manera formal a los akawaio a que solicitaran el título de sus tierras. Aunque su presentación delimitó un área de $1000 \mathrm{~km}^{2}$, el ministro redujo de manera arbitraria sus dimensiones a una cuarta parte de lo requerido. Además, el título excluyó un gran número de áreas adjudicadas con anterioridad como concesiones mineras. La población de Isseneru apeló la decisión ministerial, pero fue ignorada de nuevo. Cuando intentó impedir que los mineros provenientes de la costa y de Brasil trabajaran en sus tierras, los mineros demandaron a Isseneru y el poder judicial respaldó sus permisos, aunque nunca respondió la apelación comunal. Después de años de frustraciones, y sin esperanzas de una reparación doméstica, la población de Isseneru presentó una solicitud de medidas cautelares ante la cidh.

En respuesta al argumento del gobierno de que Isseneru no es "una comunidad akawaio tradicional" (Government of Guyana, 2013: 22), los residentes reconocen ciertos cambios: "hoy, sí, está cambiando". "En los comienzos", dijo un hombre, "no vivíamos en casas bonitas, como usted ve ahora". Por su parte, el líder electo de la comunidad, o toshao, preguntó de manera retórica: “ ¿hicimos algo malo al construir estas casas?". También culparon al gobierno por algunos de los cambios: "que el gobierno llegue a decir que no somos akawaios, cuando su escuela no enseña nuestra historia ni lengua, es hipócrita”. Otro agregó: "sabemos que estamos perdiendo nuestra cultura cuando enseñamos [a nuestros hijos] el idioma inglés únicamente. Con el desarrollo llegan los cambios". Pero otros afirmaron: "no estamos olvidando nuestras tradiciones". Respecto a los proyectos mineros y el desarrollo de la aldea, un hombre explicó:

Para ser independientes como pueblo, queremos producir nuestros propios alimentos y lo estamos haciendo. Pero también está la necesidad del dinero. Después de buscar alternativas, optamos por la minería ... Pero ... el gobierno llega con [sus] leyes y hace las cosas difíciles para nosotros.

Varias personas más explicaron su decisión de dedicarse a buscar oro. Una dijo: "con la cantidad de gente que vive en la aldea, tenemos que hacer minería". Otra agregó: "muchos foráneos hacen minería en nuestra tierra. Llegó un punto en que decidimos aprovechar estos recursos para nosotros mismos".

Migrantes de la costa de Guyana y de Brasil llegaron a buscar oro y diamantes al Mazaruni medio por primera vez en 1904. Durante la década de 1950, comenzaron a utilizar grandes dragas emplazadas en las orillas de los ríos. Desde la década de 1990, la población de Isseneru incorporó bombas a diésel y mangueras de presión, para trabajar mediante el proceso conocido como minería de chorro o hidráulica. Las cuadrillas están integradas por habitantes de

4. Notas de campo, aldea Isseneru, Guyana, 25 de noviembre de 2014. 
la aldea y trabajadores provenientes de la costa. Cada proyecto comienza a la vera de un arroyo, con el uso de las mangueras, para disolver la zona que será minada. El lodo resultante es conducido a una poza y luego bombeado con una agitadora. El último paso es amalgamar el oro con mercurio. A los trabajadores se les paga un porcentaje del producto final y todos presencian la apertura de la caja que agita el mineral y el pesaje del oro obtenido. Esta práctica es crucial, ya que la verificación mutua es lo que mantiene la confianza entre los miembros de la fuerza de trabajo étnicamente mixta.

La población de Isseneru informa que no hay mucha diferencia entre la minería que practican locales y foráneos, aunque los últimos no siempre rellenan sus pozas, como la población de Isseneru lo hace. ${ }^{5} \mathrm{El}$ Consejo de la Aldea ha emitido 25 permisos mineros a personas de Isseneru, aunque sólo siete de ellos se encontraban activos durante mi visita. El toshao se mostró preocupado porque la explotación minera regular puede provocar consecuencias negativas para la salud, además reconoció que la actividad no durará para siempre.

En la década de 1950, la población de Isseneru vivía cerca de la aldea actual y de sus actuales cultivos de yuca, cuando los mineros de oro y diamantes comenzaron a dragar el río. Para evitar potenciales conflictos, muchos se trasladaron a vivir con sus parientes al Mazaruni alto. Esta reubicación fue alentada por la administración colonial británica, que estableció escuelas y un centro de salud. A comienzos de la década de 1970, el Estado propuso construir una gran represa y una central hidroeléctrica en el Mazaruni alto (Colchester, 2005). El proyecto habría inundado todas las aldeas akawaio de la zona. En consecuencia, los pueblos del Mazaruni medio decidieron regresar a sus propias tierras y fundar la Isseneru actual.

Como subrayaron ante mí, no se trasladaron a tierras vacías ni desconocidas, regresaron a sus propias tierras, en las que sus padres y ancestros habían vivido. Una mujer dijo: "aquí los nombres de los lugares son en nuestra lengua. Por ejemplo, Apikwa [un puesto de los mineros brasileños] en realidad es Abak Ekwa [en lengua akawaio]. [El topónimo] Haimara Ekwa proviene del pez aimara.' ${ }^{6}$ Topónimos como ése confirman la antigüedad de sus reclamos de tierras. Las migraciones respondieron a las políticas coloniales, primero para evitar la llegada de mineros y después para escapar de la inundación que provocaría la represa, que los hubiera dejado sin lugar donde vivir. Su estadía en el Mazaruni alto no extinguió los derechos a la tierra en el Mazaruni medio.

Antes de solicitar al Estado el título de las tierras, el Consejo local preparó un mapa de su territorio tradicional, que incluye los recursos necesarios para el sustento de la comunidad y su modo de vida. Explicaron que no pueden cultivar en las montañas, donde el suelo es rocoso, y tampoco en suelos pantanosos.

5. Los daños en el paisaje provocados por la minería hidráulica se ven desde el aire cuando se viaja de Georgetown a Olive Creek, en el Mazaruni medio.

6. Notas de campo, Apikwa, Guyana, 28 de noviembre de 2014. 
Sólo algunas tierras son aptas para el cultivo de la yuca, que aporta el alimento y la bebida básicos. Como practican la rotación de cultivos, desplazan sus huertos cada dos o tres cosechas. Otros recursos no se encuentran disponibles en determinadas porciones de su territorio, como los árboles de corazón púrpura, cuya corteza se utiliza tradicionalmente para la fabricación de canoas, porque sólo crece hacia el sur de la aldea; tampoco las plantas de mucru con las que elaboran esos largos y angostos "exprimidores" que utilizan para procesar la yuca amarga que crece a mayor altura en el norte. La piedra que corta la yuca en rodajas está disponible sólo en las montañas. Grandes peces, entre ellos el aimara - Hoplias aimara—, se pueden pescar aguas abajo de la aldea. Muchos de estos recursos esenciales fueron excluidos del título de tierras emitido por el Estado.

Al contrario de las economías capitalistas, en las que la población satisface sus necesidades al vender su trabajo para comprar bienes, los akawaio requieren acceder a varios tipos de tierra para desarrollar la economía tradicional. En consecuencia, quienes viven en Georgetown y otras zonas costeras de Guyana no comprenden las necesidades de los akawaio ni otros pueblos amerindios. Como me explicó un experimentado abogado en Georgetown, tienden a ver sus reclamos como "acaparamiento masivo de tierras". 7 Estas miradas responden a los altos niveles de pobreza del país, que también son legado de la tradición socialista, que encuentra difícil aceptar la existencia de derechos especiales para los pueblos indígenas (Hennessy, 2012).

Los akawaio cuentan con un sistema de tenencia comunal, por el cual la tierra es propiedad de la comunidad en su conjunto y no de los individuos que la integran. Si alguien quiere establecer un huerto nuevo, él o ella son libres de limpiar la tierra siempre que no esté siendo utilizada para otro fin. Son libres de cazar y pescar en cualquier punto de sus tierras comunales. Si personas foráneas quisieran utilizar sus tierras y recursos, deben solicitar el permiso de la comunidad antes de hacerlo.

Mi llegada a Isseneru coincidió con la temporada de siembra de yuca y tuve la oportunidad de estar en una jornada de trabajo colectivo en sus nuevos cultivos. Los árboles altos ya habían sido talados y la vegetación menor removida parcialmente. El grupo cortó los árboles caídos y los almacenó junto a otras plantas secas para quemarlos después. También comenzamos a hacer pozos y sembrar ramas de yuca para el nuevo huerto. Al terminar la tarea en el terreno, se repitió el mismo proceso en el siguiente. El trabajo continuó durante varias semanas hasta que cada uno de los terrenos de la comunidad quedó listo para la siembra.

La organización colectiva del trabajo expresa el sistema comunal de tenencia de la tierra. Mientras sembrábamos la yuca, un hombre me dijo: "cuando 


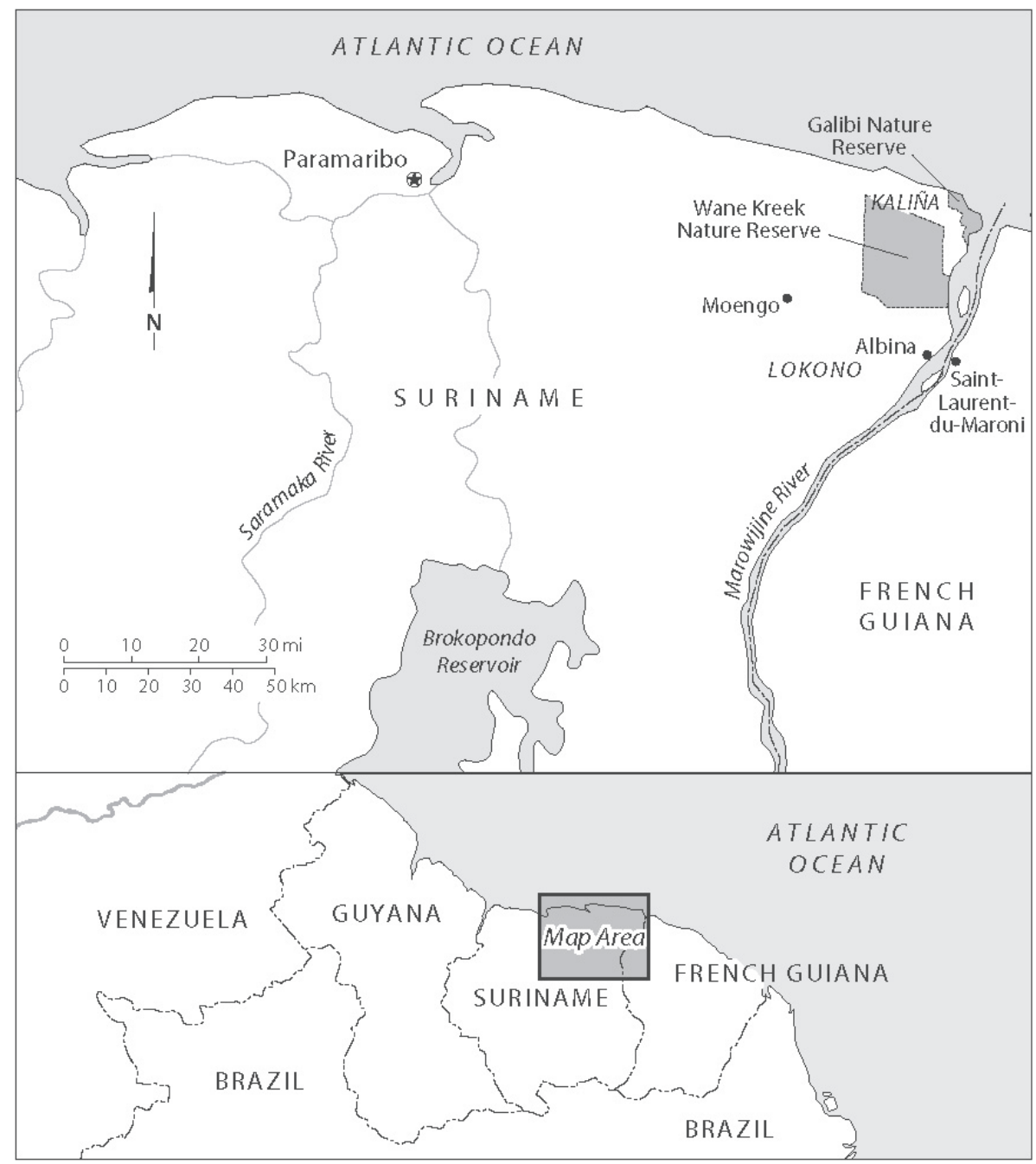

Mapa 1. Surinam (Elaboración: Bill Nelson.).

trabajo solo, me siento egoísta. Pero cuando lo hago con otros, siento que hago algo valioso". ${ }^{8} \mathrm{Su}$ afirmación de que el trabajo de mayor valor se logra mediante la colaboración, se diferencia de las sociedades capitalistas que destacan la propiedad privada mediante la aplicación del trabajo individual a la naturaleza (Locke, 1947). Entre los akawaio, el trabajo se valora por su contribución a la comunidad antes que por la acumulación individual. Sus prácticas de trabajo comunal siguen hoy tan fuertes como en el pasado y pueden contrastarse con el individualismo del empleo asalariado que domina la vida urbana en Guyana (Griffiths, 2003: 206).

8. Notas de campo, aldea Isseneru, Guyana, 26 de noviembre de 2014. 
Para los akawaio fue una sorpresa que el título que recibieron del Estado excluyera tierras que habían sido otorgadas como concesiones mineras. Las concesiones estaban protegidas por una cláusula incluida en la escritura que adjudicaba las tierras a las comunidades indígenas "salvo y excepto" si habían sido concesionadas a la minería. Como argumenta su petición a la cidh, esto contraviene principios internacionales que reconocen los derechos indígenas a la tierra como preexistentes a los Estados. Los akawaio no deberían ser privados de su propiedad como resultado de la demora estatal en reconocer sus derechos. Además, el título de sus tierras también debió ser emitido por el Estado sin impedimento alguno. Los derechos mineros podrán ser anteriores al título oficial de las tierras, pero los akawaio son preexistentes a ellos por cientos de años. Esto explica por qué muchas personas con las que hablé dijeron: "la Ley Amerindia nos traicionó". 9

Los niveles de mercurio en el Mazaruni medio son preocupantes desde la década de 1980, antes de que la población de Isseneru comenzara a explotar la minería. Se les advirtió que no consumieran mucho pescado, aunque carecen de fuentes alternativas de proteínas. Algunos mencionaron que, por los riesgos que esto implica para la salud, han intentado "reducir un poco" el consumo de pescado. Las mujeres embarazadas, en particular, fueron advertidas de no ingerirlo, pero una de ellas me preguntó: “¿qué alterativa hay?”. ${ }^{10}$

En la actualidad, el pueblo de Isseneru ejerce una influencia limitada sobre la cantidad de mercurio que se utiliza en el Mazaruni medio. La cláusula de "salvo y excepto", incluida en el título de propiedad, no sólo interfiere con la capacidad de los akawaio de controlar su territorio, sino también alienta a todos los actores de la región a considerar el Mazaruni medio como zona liberada. Investigaciones sobre la propiedad comunal han demostrado que los participantes de sistemas de acceso libre carecen del incentivo necesario para conservar los recursos y proteger el medio ambiente (Feeny et al., 1990; Ostrom, 1990), lo que puede conducir a la llamada "tragedia de los comunes" (Hardin, 1968). Por el contrario, los derechos de propiedad individuales o colectivos que incluyen el poder de excluir a otros usuarios son más propensos a reducir los impactos ambientales negativos.

Mientras los foráneos sigan accediendo a sus tierras, los akawaio no podrán limitar los efectos negativos de la minería de oro ni el uso de mercurio. Esto deja poco o nulo incentivo para restringir sus propias actividades mineras, a pesar de la preocupación por el impacto del uso del mercurio en la salud y el medio ambiente. El Estado no ha dado señales de pretender reducir el acceso al área en el futuro y continúa otorgando concesiones mineras, a pesar de su conocimiento de los problemas causados por la liberación del mercurio en el ambiente. En ausencia de nuevas medidas gubernamentales, el reconocimiento

9. Notas de campo, aldea Isseneru, Guyana, 27 de noviembre de 2014.

10. Notas de campo, aldea Isseneru, Guyana, 26 de noviembre de 2014. 
legal de los derechos indígenas a la tierra podría ayudar a controlar el uso del mercurio en el Mazaruni medio y reducir sus efectos catastróficos potenciales sobre la salud humana y el medio ambiente.

\section{Marcos interpretativos alternativos}

En ambos casos, los informes de testigo experto debían resultar legibles para múltiples audiencias, incluyendo los tres marcos superpuestos, a veces incompatibles, de los abogados y el sistema legal, las comunidades que buscan el reconocimiento de sus derechos y la antropología (Paine, 1996: 63). Para el primero, esto incluyó el uso de conceptos como "supervivencia cultural", reconocido por la cidh como derecho fundamental que debe protegerse, aunque rara vez los antropólogos hagan referencia al término, salvo en contextos de incidencia. ${ }^{11}$ Un ejemplo de la segunda categoría es mi referencia al reclamo akawaio de haber ocupado el territorio desde "tiempo inmemorial", lo cual es un modo perfectamente legítimo de referirse al pasado distante, anterior a las evidencias arqueológicas e históricas, aunque la expresión rara vez sea utilizada por los antropólogos, salvo en contextos de incidencia. Respecto al tercer marco, estos informes no son necesariamente el lugar para retomar debates teóricos sobre la compleja relación entre las organizaciones de conservación y los pueblos indígenas, aunque puede resultar útil referirse a ellas de manera selectiva.

Sin embargo, para satisfacer las expectativas de estas tres audiencias, me pareció valioso poner el énfasis en un tema prominente que surgió durante la investigación etnográfica. El hecho de centrar el foco en un tema de importancia para los integrantes de la comunidad, como la libertad o los derechos de propiedad colectivos, realza para ellos la legitimidad del informe pericial. Al desarrollar narrativas más amplias que el mero resumen de los hechos, intenté que los informes resultaran atractivos y persuasivos, atributos que los antropólogos normalmente persiguen en la escritura etnográfica. También busqué que la coherencia interna del argumento ayudara a asegurar que la evaluación general del informe no resultara afectada en la Corte, incluso si algún detalle en particular fuera cuestionado. El foco en un tema específico también ofreció la ventaja de hacer que el proyecto fuera relevante para intereses antropológicos más amplios.

11. La cidh define "supervivencia cultural" como la habilidad de "preservar, proteger y garantizar la relación especial que [ellos] tienen con su territorio", de manera que puedan "continuar viviendo su modo de vida tradicional y que su identidad cultural, estructura social, sistema económico, costumbres, creencias y tradiciones distintivas sean respetadas, garantizadas y protegidas" (cidh, 2008: $\$ \$ 37-39$ ). 


\section{Libertad en Surinam}

Para el informe pericial sobre los derechos a la tierra en Surinam, el discurso sobre la libertad fue una elección obvia. La significación de este tema fue confirmada en forma independiente por la académica surinamesa Ellen-Rose Kambel (2002: 144-150), quien identifica tres discursos utilizados por los amerindios de Surinam para cuestionar que el Estado no reconozca los derechos indígenas a la tierra: 1) el argumento de que no puede ser apropiada, que ahora parece ser un discurso viejo, dada su incompatibilidad con los objetivos políticos contemporáneos; 2) la referencia a los precedentes históricos, como el hecho de que son habitantes originales, y por lo tanto, tienen derecho a excluir a otros de su territorio, y 3) la importancia de los derechos a la tierra para su libertad. Kambel indica que sólo las dos primeras racionalidades de los derechos indígenas a la tierra fueron asumidas en los debates nacionales (2002: 154).

Además de sus referencias a la libertad de cazar y pescar en los bosques, y la necesidad de contar con derechos a la tierra para asegurar su libertad, los kaliña y lokono también mencionaron la libertad de ser indígenas al mantener su cultura y modo de vida propios. Kambel (2002) explica que los kaliña y lokono tienen conocimiento de lo establecido en la declaración de las Naciones Unidas sobre los Derechos de los Pueblos Indígenas, es decir, el "derecho colectivo a vivir en libertad, paz y seguridad como pueblos diferentes" y la libertad de expresar la "diversidad cultural indígena" sin prejuicios. En este sentido, la libertad de ser indígenas implica el derecho a determinar y reproducir importantes conocimientos y valores culturales.

El concepto de libertad conserva resonancias políticas a lo largo de Surinam, colonia holandesa entre 1667 y 1975. La mayor parte de la población del país es descendiente de esclavos o trabajadores forzados. Los areole suman 16\% de la población, pero son la facción política más fuerte. Los maroons, descendientes de los esclavos que escaparon para vivir en la selva, conforman $22 \%$ del total. El mayor grupo poblacional del país lo constituyen indios hablantes de hindi, llevados a Surinam como trabajadores forzados después de la abolición de la esclavitud, quienes comprenden $27 \%$ del total. Otro $14 \%$ de la población es descendiente de trabajadores forzados originarios de Java. Cerca de $20 \%$ se identifica como mestizo u otro. Dada la significación histórica del trabajo coercitivo en Surinam, el discurso sobre la libertad opera como poderoso unificador entre la ciudadanía y las comunidades amerindias, que comprenden entre 1.5 y $2 \%$ de la población nacional.

En estos ejemplos, la libertad es un concepto polivalente que hace referencia al mismo tiempo a ideas tradicionales sobre las personas y las relaciones sociales; la libertad de cazar y pescar en los bosques; la declaración de las 
Naciones Unidas sobre los Derechos de los Pueblos Indígenas, que garantiza la libertad de ser indígena, y el ejercicio de la libertad en un país recientemente independizado, compuesto en su mayoría por descendientes de esclavos y trabajadores forzados. La preocupación por la libertad no es sólo indígena o moderna, al mismo tiempo es compartida por los miembros del Estado y sirve de fundamento a reclamos de diferenciación.

\section{Derechos colectivos a la tierra entre los akawaio en Guyana}

El tema central del segundo informe de testigo experto, sobre el pueblo akawaio residente en la aldea de Isseneru en Guyana, fue la significación de sus derechos colectivos a la tierra como lo que los distingue de otros actores interesados en la minería de oro y diamantes en el río Mazaruni. Mi atención sobre esta pregunta fue en parte el resultado fortuito de estar presente durante la temporada de siembra de yuca, lo cual me permitió participar en una jornada de trabajo colectivo. El comentario sobre estas prácticas y su diferencia de las clásicas, liberales afirmaciones sobre la aplicación del trabajo individual a la naturaleza que resulta en la creación de propiedad privada, contribuyeron a dirigir mi atención hacia el sistema de tenencia colectiva de la tierra.

El interés en los derechos colectivos a la tierra también se vio bajo la influencia del contraste entre la Amazonía y Melanesia, donde realicé la mayor parte de mi investigación etnográfica (Kirsch, 2006). Desde la publicación del estudio pionero de Bronislaw Malinowski, Los jardines de coral y su magia (1935: 280), los antropólogos han reconocido que la tenencia de la tierra en Melanesia no es "ni lo uno ni lo otro" entre la propiedad colectiva contra la propiedad privada, "sino la relación entre reclamos colectivos y personales". Los derechos a la tierra en los ríos Ok Tedi y Fly, de Papua Nueva Guinea, donde realicé investigaciones a largo plazo, pertenecen a los miembros individuales de cada linaje y nadie tiene la autoridad para tomar decisiones sobre la tierra de otra persona (Kirsch, 2014). El contraste entre lo que me resultaba familiar de Melanesia y el énfasis en el control individual de la tierra perteneciente al linaje de los akawaio también llamó mi atención. Otra diferencia clave entre estas dos zonas culturales es que la población de Melanesia no espera acceder a todos los recursos que necesita en su propia tierra. Al contrario, valora las relaciones formadas en el intercambio, mediante las cuales adquieren recursos que de otro modo no estarían disponibles para ellos. La interdependencia con el intercambio es tan importante para la población de Melanesia como el colectivo autónomo para los akawaio. 


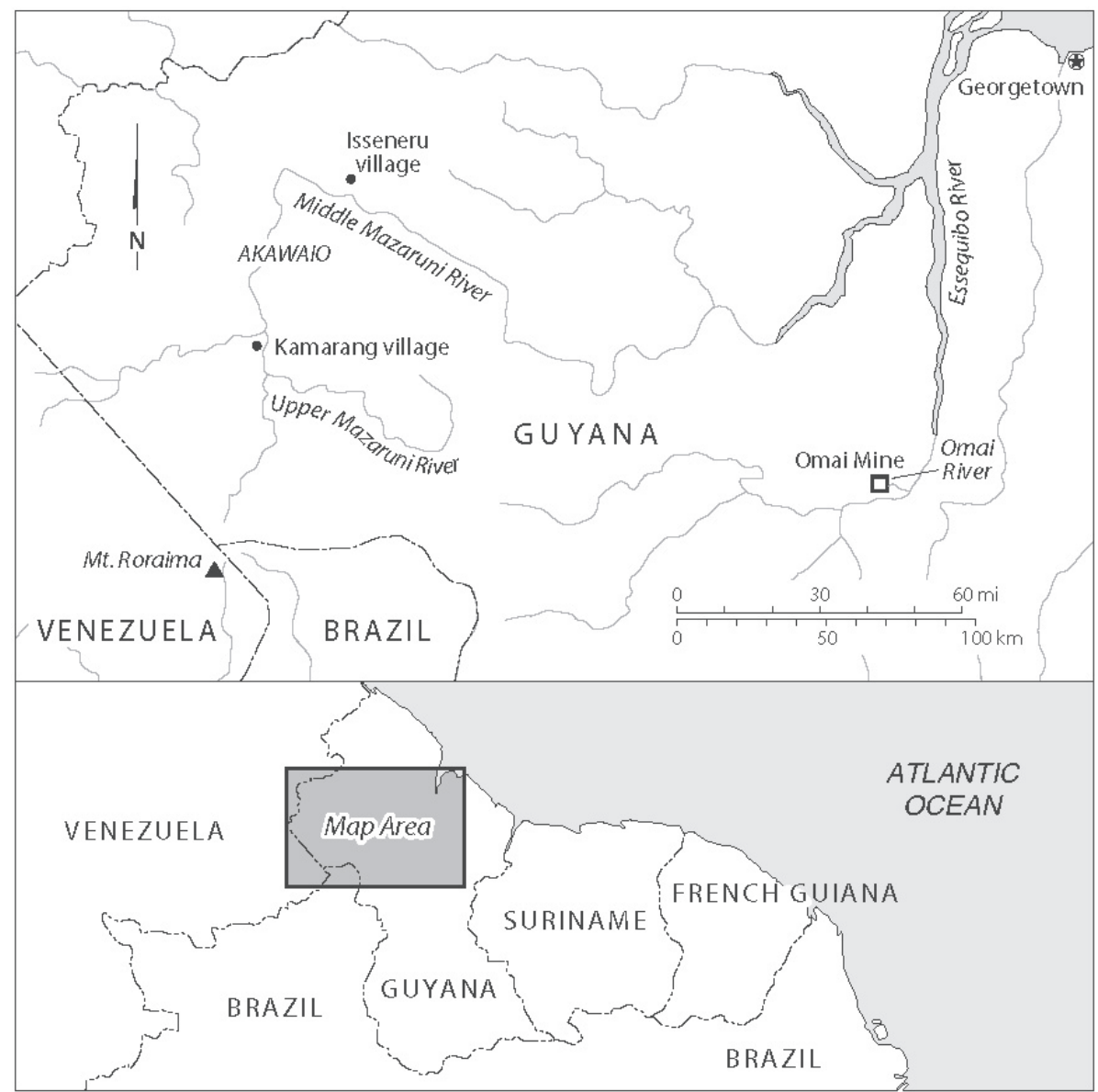

Mapa 2. Guyana (Elaboración: Bill Nelson).

\section{Desafíos de la etnografía a corto plazo}

La investigación a corto plazo sobre la que se basan los informes de testigo experto supone varios desafíos. Como mencioné, he presentado reconstrucciones estructurales de sus sistemas de tenencia de la tierra en lugar de examinar cómo sus prácticas se apartan de dichos principios generales. Por ejemplo, sobre el Mazaruni alto, el antropólogo Tom Griffiths (2003: 6) apunta que "hallazgos orales y empíricos confirman que [ . . ] la colectividad conserva derechos de propiedad sobre el territorio total [ . . . ] mientras que las comunidades, familias e individuos poseen diferentes niveles de tenencia subsidiaria y derechos de uso sobre porciones de ese territorio". También es probable que la experiencia histórica de inseguridad en los derechos a la tierra haya contribuido a la reificación de su sistema de tenencia comunal, en contraste, por ejemplo, con la atrofia del compromiso ideológico de los sistemas de propiedad comunal de 
tierras que Gabriela Torres-Mazuera (2016) describe para el sistema de ejidos en México, resultante de la modificación y privatización gradual de las tierras comunales. Para los fines del informe de testigo experto, sin embargo, fue suficiente describir el carácter general de esos arreglos, en especial debido a mi mandato más amplio de examinar el impacto de la minería de oro en el uso de la tierra, el proceso de titulación del Estado y otras preocupaciones señaladas en la petición a la Comisión Interamericana de Derechos Humanos.

Otra diferencia clave entre el presente texto y otras investigaciones etnográficas más tradicionales es mi dependencia del informe oral. Los antropólogos reconocemos la distancia entre lo que las personas dicen y lo que en efecto hacen. Durante el desarrollo del trabajo de campo, es posible, por lo general, hacer las correcciones necesarias mediante la observación directa o la confirmación independiente por parte de otros miembros de la comunidad. Por el contrario, no me fue posible documentar denuncias específicas de avances sobre tierras indígenas, incluso habiendo recibido informes de múltiples actores. Tampoco tuve oportunidad de completar las historias orales con investigación de archivo. El reporte directo de lo que la población me dijo atribuye a sus comentarios el mismo peso que hechos confirmados, incluso cuando no siempre sean equivalentes.

Otro desafío de la investigación a corto plazo es lograr el tipo de relación que normalmente se establece con los informantes durante el curso de trabajos de campo más extensos. Un indicador de la calidad de mis relaciones con la población de Surinam y Guyana fue que todas nuestras reuniones se llevaron a cabo en espacios públicos, pues no fui invitado a ninguna casa. Aunque encontramos oportunidades para interactuar durante la noche en la ciudad de Albina, en Surinam (Geertz, 1998), o para tomar cerveza Banks y bailar tecno-pop brasileño a todo volumen en un pequeño puesto minero sobre el río Mazaruni, la mayor parte de nuestro tiempo juntos fue bastante estructurado. No obstante, en ambos casos pude ganarme su confianza y lograr que accedieran a mi pedido de escribir sobre su situación, a pesar de las críticas a los antropólogos.

\section{Dilemas políticos: mercurio y medio ambiente}

Escribir informes de testigo experto para apoyar demandas legales también puede colocar a los antropólogos ante dilemas políticos. ${ }^{12}$ Una primera preocupación es que los argumentos de estos informes podrían influir en la respuesta que se dé a futuros reclamos de derechos indígenas. Como Charles Hale se

12. En su discusión sobre el caso del pueblo saramaka vs. Surinam (cidh, 2008), Richard Price (2011: 238-239) describe la "tensión fundamental" entre la reificación de la cultura y la tradición en el derecho internacional de los derechos humanos y el discurso antropológico contemporáneo. 
pregunta: “¿cómo formular reclamos indígenas a la tierra y representarlos en el lenguaje necesario para alcanzar su reconocimiento legal, sin mostrarlos en términos que reforzarían rigideces internas ni crear criterios que otras comunidades subalternas serían incapaces de alcanzar?” (2006: 11). Es posible, por ejemplo, que los argumentos sobre derechos colectivos a la tierra se apliquen como prueba de fuego para los derechos indígenas en Guyana o cualquier otro lugar, a pesar de la diversidad de regímenes de propiedad indígena y su flexibilidad para responder al cambio político y económico. A pesar de ello, elegí enfocarme en los derechos colectivos a la tierra porque la población de Isseneru siente que la define y a la vez la distingue de otros grupos étnicos en Guyana.

También está el riesgo de generar reacciones políticas al reproducir representaciones esencializadas de las actitudes indígenas hacia el medio ambiente (Conklin, 1997; Redford, 1991). En el caso de Surinam, el capitán Pané rescató la contribución del conocimiento y las prácticas tradicionales para proteger a las tortugas de mar en Galibi, a pesar de su anterior participación en la venta de los huevos. De modo similar, Jérémie Gilbert y Victoria Tauli-Corpuz dieron testimonio de la compatibilidad de los derechos indígenas a la tierra y la conservación de la biodiversidad, a pesar de lo que los antropólogos saben sobre las actitudes de los pueblos indígenas hacia el medio ambiente y el desarrollo (Kirsch, 1997; 2007). El rechazo de estos estereotipos es evidente en el caso de Guyana, donde los akawaio son criticados por practicar la minería artesanal de oro, que provoca significativos impactos ambientales. Del mismo modo, la idea popular, en Guyana, de que los amerindios están interesados en extender sus derechos a la tierra mientras contengan reservas probadas reduce cínicamente la cuestión de los derechos indígenas a la contienda política por obtener más derechos mineros (Hilson y Laing, 2017b: 180). Los esfuerzos por justificar reclamos de tierras con referencia al deseo de "continuar nuestros modos de vida tradicionales" rebotaron contra los amerindios involucrados en la minería de oro, en especial porque la minería tiende a producir impactos negativos en las actividades económicas tradicionales (Hilson y Laing, 2017b: 177). Sin embargo, la población de Isseneru intentaba proteger sus derechos a la tierra por diversos motivos, que incluyen la importancia del trabajo colectivo por razones sociales. Explotaban el oro, pero también necesitaban tierras para sembrar la yuca y obtener recursos considerables.

También era importante reconciliar el apoyo a su reclamo por los derechos a la tierra con las preocupaciones por el uso del mercurio. La contaminación producida por la amalgama del oro provoca impactos ambientales que implican serios riesgos para la salud humana. Cuando estuve en la capital Georgetown, entrevisté a un biólogo pesquero de la Universidad de Guyana, cuyo primer proyecto de investigación fue estudiar el impacto del infame derrame de cianuro ocurrido en la mina de oro Omai, en 1995, que extinguió de manera temporal toda la vida orgánica en el río del mismo nombre, un tributario del 
Essequibo, el más importante del país. Cuando le pregunté qué le preocupaba más, si los impactos del cianuro o el mercurio, ambas sustancias utilizadas para el procesamiento del oro, su respuesta me sorprendió. Me explicó que, en los trópicos, el cianuro se diluye en periodos relativamente cortos, en consecuencia, el río Omai recuperó su fauna ictícola y vegetal proveniente de cursos de agua adyacentes más rápido de lo que se creía. Por el contrario, el mercurio se acumula en la cadena trófica y el proceso de metilación que lo vuelve biodisponible ocurre con rapidez en los ríos de las tierras bajas de Guyana, que con frecuencia inundan sus orillas y generan pozas que facilitan ese tipo de reacciones. En consecuencia, el biólogo pesquero estaba mucho más preocupado por los efectos a largo plazo del mercurio utilizado en la minería de oro artesanal, a pesar de haber sido testigo de uno de los peores derrames de cianuro en la historia de la minería industrial (entrevista con ecologista de agua dulce, Georgetown, Guyana, 5 de diciembre de 2014).

En un principio, supuse que para escribir un informe de testigo experto en apoyo a los derechos a la tierra del pueblo akawaio debería suspender mi juicio personal sobre la cuestión de la contaminación por mercurio, pero durante el proceso de conversación con la población de Isseneru, aprendí que ellos comparten la preocupación sobre los impactos del mercurio en el medio ambiente, la salud y el bienestar. Pronto reconocí que sólo con pleno control sobre su territorio, y con la potestad de excluir a otros mineros artesanales, los akawaio residentes en Isseneru serán capaces de limitar los impactos ambientales del mercurio en el futuro.

\section{Conclusión: ¿informes de testigo experto como etnografía "suficiente"?}

Por último, es razonable preguntarse si estos informes, basados en investigación comprometida a corto plazo, califican como etnografía "suficiente" (Scheper-Hughes, 1989: 28). Gaynor Macdonald (2002: 107) sostiene que la investigación instrumental llevada a cabo en apoyo a demandas legales es incompatible con el ideal disciplinario de textos etnográficos con final abierto, capaces de admitir interpretaciones opuestas. Sin embargo, los proyectos de investigación a corto plazo, como los ejemplos que aquí se plantearon, están tal vez más cerca del enfoque centrado en la resolución de problemas de la mayor parte de la etnografía contemporánea, que al tipo de "descripción densa" que antes dominaba la disciplina (Geertz, 1973). Estos proyectos tienen en común más de lo que podría suponerse con trabajos de colegas que hacen antropología jurídica en otros contextos, estudios sobre las corporaciones, ciencia y tecnología, dada su duración reducida y orientación pragmática. No obstante, los hallazgos etnográficos de estos proyectos a corto plazo no pueden separarse 
de los contextos en los que fueron producidos. Sin embargo, lo que aprendí durante las entrevistas y grupos de discusión se vio bajo la influencia inevitable del proceso legal. Por ejemplo, mis informantes en Surinam y Guyana recalcaron las consecuencias negativas de la imposibilidad de excluir a otros de su propia tierra porque ése era el foco de su demanda. Por lo tanto, la información reunida para los informes periciales no es lo suficientemente robusta como para presentarse por sí misma, sin importar el contexto en el que fue producida (Macdonald, 2002). No obstante, ambos casos ofrecen lecciones significativas y comparables sobre cómo se formulan y discuten los reclamos por derechos indígenas a la tierra, así como las formas de adjudicación que atienden dichos reclamos, lo que incluye cómo los discursos sobre la libertad o los derechos indígenas a la tierra son movilizados por actores diversos en diferentes contextos nacionales.

\section{Bibliografía}

African Commission on Human and Peoples' Rights (achpr), 2009, “276/03 Centre for Minority Rights Development (Kenya) and Minority Rights Group (on Behalf of Endorois Welfare Council)/Kenya”, 46th Ordinary Session, del 11 al 25 de noviembre, Gambia. Disponible en línea: <http://www.achpr.org/files/ sessions/46th/comunications/276.03/achpr46_276_03_eng.pdf >.

Butt Colson, Audrey J., 2009, Land: Its Occupation, Management, Use and Conceptualization. The Case of the Akawaio and Arekuna of the Upper Mazaruni Distinct, Guyana, Last Refuge, Panborough.

Colchester, Marcus, 2004, "Conservation Policy and Indigenous Peoples", en Environmental Science \& Policy, vol. 7, núm. 3, pp. 145-153.

— 2005, "Maps, Power, and the Defence of Territory: The Upper Mazaruni Land Claim in Guyana", en J. Peter Brosius, Anna Lowenhaupt Tsing y Charles Zerner (eds.), Communities and Conservation: Histories and Politics of Community-Based Natural Resource Management, Alta Mira, Walnut Creek, pp. 271-303.

Commissie Landrechten Inheemsen Beneden-Marowijne (clim), 2006, "Marauny Na'na Emandobo Lokono Shikwabana. Marowijne, Our Territory. Traditional Use and Management of the Lower Marowijne Area by the Kaliña and Lokono", Vereniging van Inheemse Dorpshoofden in Suriname/Forest Peoples Programme, Paramaribo.

Conklin, Beth, 1997, "Body Paint, Feathers, and vcrs: Aesthetics in Authenticity in Amazonian Activism”, en American Ethnologist, vol. 24, núm. 4, pp. 711-737.

Corte Interamericana de Derechos Humanos (cidh), 2008, "Caso del pueblo saramaka vs. Surinam. Sentencia de 12 de agosto de 2008 (interpretación de la sentencia de excepciones preliminares, fondo, reparaciones y costas)", serie C, núm. 185. Disponible en línea: <http://www.corteidh.or.cr/docs/casos/articulos/seriec_185_ esp.pdf>.

— 2015, "Caso pueblos kaliña y lokono vs. Surinam, sentencia de 25 de noviembre de 2015 (fondo, reparaciones y costas)", serie C, núm. 309. Disponible en línea: $<$ http://www.corteidh.or.cr/docs/casos/articulos/seriec_309_esp.pdf >. 
Feeny, David, Fikret Berkes, Bonnie J. McCay y James M. Acheson, 1990, “The Tragedy of the Commons: Twenty-Two Years Later", en Human Ecology, vol. 18, núm. 1, pp. 1-19.

Geertz, Clifford, 1973, The Interpretation of Cultures: Selected Essays, Basic Books, Nueva York.

— 1998, “Deep Hanging Out”, en The New York Review of Books, vol. 45, núm. 16, p. 69.

Goodland, Robert (ed.), 2009, Suriname's Bakhuis Bauxite Mine: An Independent Review of srk's Impact Assessment, Vereniging van Inheemse Dorpshoofden in Suriname, Paramaribo.

Government of Guyana, 2013, "Response to the Request for Precautionary Measures. Request for Information. Re: Akawaio Indigenous Community of Isseneru and the Amerindian Peoples' Association (apa)", presentado a la Comisión Interamericana de Derechos Humanos, MC-283-13, 30 de noviembre.

Griffiths, Tom, 2003, “Ownership, Use, Occupation and Management of Ancestral Akawaio and Arekuna Territory in the Upper Mazaruni, Guyana: A Study of Traditional Tenure over Lands and Territory Covered by the 1959 Upper Mazaruni Amerindian District", informe pericial sobre tenencia de la tierra.

Hale, Charles R., 2006, "Activist Research v. Cultural Critique: Indigenous Land Rights and the Contradictions of Politically Engaged Anthropology", en Cultural Anthropology, vol. 21, núm. 1, pp. 96-120.

Hardin, Garrett, 1968, “The Tragedy of the Commons”, en Science, vol. 62, núm. 3859, pp. 1243-1248.

Hennessy, Logan, 2012, "Re-Placing Indigenous Territory: Villagization and the Transformation of Amerindian Environments Under 'Cooperative Socialism' in Guyana”, en Annals of the Association of American Geographers, vol. 103, núm. 5, pp. 1242-1265.

Hilson, Gavin y Tim Laing, 2017a, “Guyana Gold: A Unique Resource Curse?”, en The Journal of Development Studies, vol. 53, núm. 2, pp. 229-248.

_ 2017b, "Gold Mining, Indigenous Land Claims and Conflict in Guyana's Hinterland”, en Journal of Rural Studies, vol. 50, pp. 172-187.

Kambel, Ellen-Rose, 2002, "Resource Conflicts, Gender, and Indigenous Rights in Suriname: Local, National and Global Perspectives", tesis de doctorado, Universidad de Leiden, Leiden.

Kirsch, Stuart, 1997, "Regional Dynamics and Conservation in Papua New Guinea: The Lakekamu River Basin Project", en The Contemporary Pacific, vol. 9, núm. 1, pp. 97-121.

- 2006, Reverse Anthropology: Indigenous Analysis of Social and Environmental Relations in New Guinea, Stanford University Press, Stanford.

— 2007, "Indigenous Movements and the Risks of Counterglobalization: Tracking the Campaign against Papua New Guinea’s Ok Tedi Mine”, en American Ethnologist, vol. 34, núm. 2, pp. 303-321.

— 2009, "Comments on the Bakhuis Draft Environmental and Social Impact Report", en Robert Goodland (ed.), Suriname's Bakhuis Bauxite Mine: An Independent Review of srk's Impact Assessment, Vereniging van Inheemse Dorpshoofden in Suriname, Paramaribo, pp. 26-59.

— 2014, Mining Capitalism: The Relationship between Corporations and their Critics, University of California Press, Berkeley. 
Locke, John, 1947 [1689], Two Treatises of Government, Hafner, Nueva York.

Macdonald, Gaynor, 2002, "Ethnography, Advocacy and Feminism: A Volatile Mix. A View from a Reading of Diane Bell's Ngarrindjeri Wurruwarrin", en The Australian Journal of Anthropology, vol. 13, núm. 1, pp. 88-110.

Malinowski, Bronislaw, 1935, Coral Gardens and their Magic: A Study of the Methods of Tilling the Soil and of Agricultural Rites in the Trobriand Islands, Routledge, Londres.

Ostrom, Elinor, 1990, Governing the Commons: The Evolution of Institutions for Collective Action, Cambridge University Press, Cambridge.

Paine, Robert, 1996, "In Chief Justice McEachern's Shoes: Anthropology's Ineffectiveness in Court", en Political and Legal Anthropology Review, vol. 19, núm. 2, pp. 59-70.

Price, Richard, 2011, Rainforest Warriors: Human Rights on Trial, University of Pennsylvania Press, Filadelfia.

Redford, Kent H., 1991, “The Ecologically Noble Savage”, en Cultural Survival Quarterly Magazine, vol. 15, núm. 1, pp. 46-48.

Scheper-Hughes, Nancy, 1989, Death without Weeping: The Violence of Everyday Life in Brazil, University of California Press, Berkeley.

Torres-Mazuera, Gabriela, 2016, "Deregulating the Social Life of Property: Neoliberalism and the Proliferation of Normative Dissonances in Mexico", en The Journal of Legal Pluralism and Unofficial Law, vol. 48, núm. 1, pp. 58-74.

Vereniging van Inheemse Dorpshoofden in Suriname (vids), 2009, "Securing Indigenous Peoples' Rights in Conservation in Suriname: A Review", Vereniging van Inheemse Dorpshoofden in Suriname/Forest Peoples Programme, Moreton-inMarsh.

\section{Entrevistas}

Ricardo Pané, Galibi, Surinam, 19 de enero de 2009.

Informante, Wan Shi Sha, aldea Marijkedorp, Surinam, 18 de enero de 2009.

Ecologista de agua dulce, Georgetown, Guyana, 5 de diciembre de 2014.

*Agradezco a Luis Manuel Claps por traducir este texto y a Bill Nelson por los mapas. También a los coordinadores de este número, Rosalva Aída Hernández Castillo, Christopher Loperena y Mariana Mora, por la invitación a participar en el proyecto y su acompañamiento. Gracias a los participantes del Taller de Peritajes Antropológicos, en el Centro de Investigaciones y Estudios Superiores-Ciudad de México, por su retroalimentación a mi presentación, al Centro de Estudios de América Latina y el Caribe de la Universidad de Michigan por mi viaje, al Programa "Translating the Americas" y al Departamento de Antropología de la Universidad de Michigan por su apoyo con la traducción, y a los revisores anónimos por sus constructivas sugerencias. 